\title{
Large deviations of ergodic counting processes: a statistical mechanics approach
}

\author{
Adrián A. Budini \\ Consejo Nacional de Investigaciones Científicas y Técnicas, \\ Centro Atómico Bariloche, Avenida E. Bustillo Km 9.5, (8400) Bariloche, Argentina
}

(Dated: September 17, 2018)

\begin{abstract}
The large-deviation method allows to characterize an ergodic counting process in terms of a thermodynamic frame where a free energy function determines the asymptotic non-stationary statistical properties of its fluctuations. Here, we study this formalism through a statistical mechanics approach, i.e., with an auxiliary counting process that maximizes an entropy function associated to the thermodynamic potential. We show that the realizations of this auxiliary process can be obtained after applying a conditional measurement scheme to the original ones, providing is this way an alternative measurement interpretation of the thermodynamic approach. General results are obtained for renewal counting processes, i.e., those where the time intervals between consecutive events are independent and defined by a unique waiting time distribution. The underlying statistical mechanics is controlled by the same waiting time distribution, rescaled by an exponential decay measured by the free energy function. A scale invariance, shift closure, and intermittence phenomena are obtained and interpreted in this context. Similar conclusions apply for non-renewal processes when the memory between successive events is induced by a stochastic waiting time distribution.

PACS numbers: 05.70.Ln, 05.40.-a, 42.50.Lc, 02.50.-r
\end{abstract}

\section{INTRODUCTION}

In different scientific disciplines the measurement realizations of a given process consist of a set of random points distributed along the real number line. These "stochastic point processes" [1-4] can be characterized through different statistical objects and techniques. One of the most usual is to count the (random) number of events up to a given point. For example, one can count up to a given time the number of photons emitted by a quantum optical system [5-13], the number of electrons transported through a nanoscopic structure [14 16], or the number of spikes produced by a neuronal system 17 21], just to name a few. From these examples it becomes clear that the underlying (non-equilibrium) dynamics that lead to the random processes may be of very different nature and complexity.

A counting process can statistically be characterized by a set of functions, each one defining the probability of n-events occurring up to a given time. These objects completely characterize the fluctuations (moments and cumulants) of the measurement realizations. The behavior (time-dependence) of the counting probabilities is not universal and depends on each specific case. Nevertheless, after a characteristic transient time that depends on each system, a general characterization is expected to apply. In fact, when the probabilities of a system scales as exponential functions (asymptotic regime), the large deviation (LD) approach [22] allows to read the scaling rates in the context of an equilibrium thermodynamics formalism. In this frame [22 29], the entire (asymptotic) statistical properties of a process can be obtained from a free energy function [30] and characterized, in particular, from its associated thermodynamic response functions (dynamical order parameters), i.e., its derivatives with respect to an intensive parameter (conjugate field). The thermodynamic frame describes in a unified way both the typical fluctuations of a system, such as those that can be fit in a Gaussian description [central limit theorem (CLT)], but also large deviation fluctuations that go beyond the previous regime.

The LD theory and its associated thermodynamic frame were recently applied for characterizing photon counting processes in different quantum optical systems [28, 29]. The thermodynamic responses develop properties such as scale invariant points, phase transitions and finite-size effects, which in turn indicate drastic changes in the statistical properties of the counting process.

While previous results [28, 29] confirm that the LD method provides a deep description of a stochastic counting process, its associated thermodynamic structure can only be analyzed after specifying a given problem or situation. Furthermore, while the full thermodynamic formalism depends on the conjugate (counting) field it is not completely clear how this dependence may become observable or explicitly defined from the statistics of the counting process. The main goal of this paper is to shed light on this issue and to demonstrate that some general features of the thermodynamic frame can be characterized without knowing the specific properties and underlying dynamics (classical, quantum, phenomenological) of the counting process. The present analysis applies to ergodic (unidirectional) processes.

Instead of focusing on the thermodynamic properties, which depend on each particular problem, here we analyze its associated statistical mechanics, i.e., an auxiliary counting process that maximizes an entropy function related to the free energy of the thermodynamic frame. We show that its realizations can be obtained after applying a conditional measurement scheme (Fig. 1) to the original ones. The role played by the conjugate field is clarified in this context, providing an alternative measurement foun- 
dation of the formalism and results developed in Refs. [28, 29]. Moreover, some general results and conclusions follow from the measurement scheme.

A general analysis is developed by assuming a renewal property [1-4]. Hence, the counting process is characterized by a probability distribution, or waiting time distribution (WTD), that defines the statistics of the random time intervals between consecutive events. On the basis of the conditional measurement scheme, we show that the thermodynamic frame can also be related to an underlying renewal counting process whose WTD is the original one multiplied by an exponential decay scaled by the free energy function. Furthermore, it is demonstrated that scale invariant points, a shift closure property, intermittent and (thermodynamic) finite-size effects can be developed under the renewal hypothesis. While no general results can be formulated for non-renewal counting process, we show that similar conclusions can be obtained for process defined by a stochastic WTD. This non-renewal case arises in different situations such as quantum optical systems [13] and neuronal ones [17.

The paper is outlined as follows. In Sec. II, after reviewing the LD approach in the context of counting processes, we define and analyze the underlying statistical mechanics associated to the thermodynamic formalism. In Sec. III we study the central case of renewal counting process. In Sec. IV we analyze non-renewal processes defined by a stochastic WTD. In Sec. V we provide the conclusions.

\section{LARGE DEVIATION THEORY OF COUNTING PROCESSES}

A counting process can be statistically characterized by a set of probabilities $\left\{P_{n}(t)\right\}_{n=0}^{\infty}$, satisfying $0 \leq P_{n}(t) \leq$ 1 , and the normalization $\sum_{n=0}^{\infty} P_{n}(t)=1$. Each $P_{n}(t)$ is the probability of occurrence of $n$-events up to time $t$. As in Refs. 28, 29], here we are restricting our analysis to unidirectional counting processes, i.e., $n \geq 0$.

While the short time behavior of the counting probabilities depends on each specific case, their asymptotic behavior may assume a universal structure. For example, CLT allows to approximate their long time regime with a Gaussian distribution [2, 3]

$$
\lim _{t \rightarrow \infty} P_{n}(t) \stackrel{c l t}{\approx} \sqrt{\frac{1}{2 \pi \overline{\Delta n_{t}^{2}}}} \exp \left\{-\frac{\left[n-\bar{n}_{t}\right]^{2}}{2 \overline{\Delta n_{t}^{2}}}\right\},
$$

where $\bar{n}(t)$ is the average number

$$
\bar{n}_{t}=\sum_{n=0}^{\infty} n P_{n}(t) \approx\langle\langle n\rangle\rangle t,
$$

while $\overline{\Delta n_{t}^{2}}$ corresponds to the second cumulant

$$
\overline{\Delta n_{t}^{2}}=\sum_{n=0}^{\infty}(n-\bar{n}(t))^{2} P_{n}(t) \approx\left\langle\left\langle\Delta n^{2}\right\rangle\right\rangle t .
$$

$\langle\langle n\rangle\rangle$ and $\left\langle\left\langle\Delta n^{2}\right\rangle\right\rangle$ are the asymptotic growing rates. For a fixed time, the prediction of CLT is only valid up to a given accuracy in a central region of the counting probabilities. By assuming valid an exponential asymptotic behavior

$$
\lim _{t \rightarrow \infty} P_{n}(t) \approx \exp \left[-t \varphi\left(\frac{n}{t}\right)\right]
$$

LD method allows to describe the long time regime beyond the Gaussian approximation. On the basis of a saddle-point approximation [22], the function $\varphi(n)$ can be obtained from a Legendre-Fenchel transformation

$$
\varphi(n)=\max _{s}[\Theta(s)-s n],
$$

together with the inversion formula

$$
\Theta(s)=\min _{n}[\varphi(n)+s n] .
$$

The function $\Theta(s)$ is defined by the asymptotic behavior

$$
\lim _{t \rightarrow \infty} Z(t, s) \approx \exp [-t \Theta(s)]
$$

Here, $Z(t, s)$ is the characteristic function associated to the counting probabilities

$$
Z(t, s) \equiv \sum_{n=0}^{\infty} P_{n}(t) e^{-s n},
$$

where $s$ is a real dimensionless parameter. Hence, $\Theta(0)=$ 0 . From relations (5) and (6) one deduces that any of the functions $\varphi(n)$ or $\Theta(s)$ provide a complete description of the asymptotic counting statistics.

CLT is covered by the LD approach. From Eq. (1) the LD rate function $\varphi(n)$ can be approximated as

$$
\varphi(n) \stackrel{c l t}{\approx} \frac{(n-\langle\langle n\rangle\rangle)^{2}}{2\left\langle\left\langle\Delta n^{2}\right\rangle\right\rangle} .
$$

Furthermore, Eq. (6) allows us to obtain

$$
\Theta(s) \stackrel{\text { clt }}{\approx} s\langle\langle n\rangle\rangle-\frac{s^{2}}{2}\left\langle\left\langle\Delta n^{2}\right\rangle\right\rangle
$$

This expression can be read as a Taylor expansion up to second order in $s$ of $\Theta(s)$, which in turn corresponds to a quadratic fitting of $\varphi(n)$ around its minimal value, $n=$ $\langle\langle n\rangle\rangle$. Thus, the Gaussian prediction of CLT follows from a quadratic approximation of both $\varphi(n)$ and $\Theta(s)$. The LD method takes into account all higher contributions related to higher cumulants.

\section{A. Underlying thermodynamics and statistical mechanics}

Legendre-Fenchel transformations (5) and (6) suggest to analyze the LD method within an equilibrium thermodynamic frame [28, 29]. In fact, the functions $\varphi(n)$ 
and $\Theta(s)$ can be read and related to the internal energy $(U)$ and grand potential $[\Theta(s)]$ of a thermodynamic open system, which satisfy the relation [30]

$$
\Theta(s)=U-T S+s N_{\#} .
$$

$N_{\#}$ is the average "particle number," $s$ plays the role of a (dimensionless) chemical potential (intensive parameter), and the (dimensionless) temperature $T$ can be taken as one. Here, we re-derive these relations and associations [29] from an equivalent statistical mechanics approach. Hence, we search for a set of auxiliary (counting) probabilities $\left\{q_{n}(t)\right\}_{n=0}^{\infty}$ that maximize an entropy function $S$ consistent with the thermodynamic relation (11).

As the LD approach applies in the asymptotic regime, we define a normalized entropy function as

$$
S \equiv-\lim _{t \rightarrow \infty} \frac{1}{t} \sum_{n=0}^{\infty} q_{n}(t) \log \left[q_{n}(t)\right]
$$

In order to find the probabilities $\left\{q_{n}(t)\right\}_{n=0}^{\infty}$, we maximize the entropy function (at a fixed time) under the constraints of probability normalization $\sum_{n=0}^{\infty} q_{n}(t)=1$, fixed average (particle) number

$$
N_{\#}=\langle\langle N\rangle\rangle \equiv \lim _{t \rightarrow \infty} \frac{1}{t} \sum_{n=0}^{\infty} q_{n}(t) n,
$$

and (average) internal energy

$$
U=\langle\langle E\rangle\rangle \equiv-\lim _{t \rightarrow \infty} \frac{1}{t} \sum_{n=0}^{\infty} q_{n}(t) \log \left[P_{n}(t)\right] .
$$

By using the method of Lagrange multipliers [30], after defining the free energy or grand potential [see Eq. (7)]

$$
\Theta(s)=-\lim _{t \rightarrow \infty} \frac{1}{t} \log [Z(t, s)],
$$

and using the thermodynamic relation Eq. (11), we get the asymptotic behavior

$$
\lim _{t \rightarrow \infty} q_{n}(t) \approx \exp \left\{-t\left[\left(\frac{n}{t}\right) s+\varphi\left(\frac{n}{t}\right)-\Theta(s)\right]\right\} .
$$

In this derivation, we assumed units of energy such that the Lagrange multiplier associated to the internal energy constraint (inverse of the temperature) takes the value one $(T \rightarrow 1)$, and $s$ corresponds to the Lagrange multiplier associated to the constraint (13), i.e., the dimensionless parameter $s$ plays the role of a chemical potential.

Eq. (16) is satisfied if we define the probabilities $q_{n}(t, s) \equiv q_{n}(t)$ at any time as [see Eqs. (4) and (7)]

$$
q_{n}(t, s) \equiv \frac{1}{Z(t, s)} P_{n}(t) e^{-s n},
$$

where the "partition function" $Z(t, s)$ follows from Eq. (8). These probabilities define an extra counting process, which is parametrized by the dimensionless chemical potential $s$. Due to this dependence, the set of stochastic realizations consistent with $\left\{q_{n}(t, s)\right\}_{n=0}^{\infty}$ is named as the $s$-ensemble 28, 29].

Relevant information about the fluctuations of the counting process defined by the set $\left\{P_{n}(t)\right\}_{n=0}^{\infty}$ is encoded in the thermodynamical response functions [30], such as the first and second derivatives of the grand potential with respect to $s$

$$
\langle\langle N\rangle\rangle=\frac{\partial}{\partial s} \Theta(s), \quad\left\langle\left\langle\Delta N^{2}\right\rangle\right\rangle=-\frac{\partial^{2}}{\partial s^{2}} \Theta(s) .
$$

In fact, different (dynamical) phases, as well as thermodynamic transitions between them, can be established by analyzing the dependence of these objects with respect to the pseudo chemical potential [28, 29]. Due to this reason, the first response function is alternatively denoted as a "dynamical order parameter," while $s$ is quoted as its non-equilibrium "conjugate (counting) field" [28].

Response functions (18) can be written as the normalized $\left[\lim _{t \rightarrow \infty}(1 / t) \cdots\right]$ average value and variance of the number of events associated to the set of probabilities $\left\{q_{n}(t, s)\right\}_{n=0}^{\infty}$ [see Eq. (13)]. The same property is valid for higher objects. Hence, the $s$-ensemble provides an alternative basis for describing the thermodynamic frame. This fact defines the present approach.

\section{B. Realizations of the s-ensemble: conditional counting scheme}

The point process associated to probabilities $\left\{P_{n}(t)\right\}_{n=0}^{\infty}$ is our observable system. Therefore, one has access to its ensemble of realizations. Nevertheless, it is not clear which kind of point process leads to the counting process defined by the probabilities $\left\{q_{n}(t, s)\right\}_{n=0}^{\infty}$, Eq. (17). While its definition seems to be rather abstract, here we provide a measurement scheme for generating its ensemble of realizations from those of $\left\{P_{n}(t)\right\}_{n=0}^{\infty}$. The dependence of the thermodynamic frame on the conjugate field $s$ is clarified by this result, which in turn answers the main issue raised up in the introduction.

When $s>0$, we realize that $0<\exp [-s]<1$. Therefore, this factor can be read as a probability. In this case, the realizations associated to the counting probabilities $\left\{q_{n}(t, s)\right\}_{n=0}^{\infty}$ in the interval $(0, t)$ can be obtained as follows. Each realization corresponding to $\left\{P_{n}(t)\right\}_{n=0}^{\infty}$ is split in two channels (upper and lower arms, see Fig. 1) with probabilities $\exp [-s]$ and $(1-\exp [-s])$ respectively. If in the interval $(0, t)$ all events are selected in the upper arm, that realization is taken as one of the set $\left\{q_{n}(t, s)\right\}_{n=0}^{\infty}$ [Fig. 1(a)]. If at least one event happens in the lower arm, the realization is discarded [Fig. 1(b)]. Therefore, each realization of $\left\{P_{n}(t)\right\}_{n=0}^{\infty}$ having $n$-events is selected as one of the $\left\{q_{n}(t, s)\right\}_{n=0}^{\infty}$ with probability $\exp [-s n]$ and discarded with probability $\sum_{k=1}^{n}\left(\begin{array}{l}n \\ k\end{array}\right) e^{-s(n-k)}\left(1-e^{-s}\right)^{k}=\left(1-e^{-s n}\right)$. This con- 

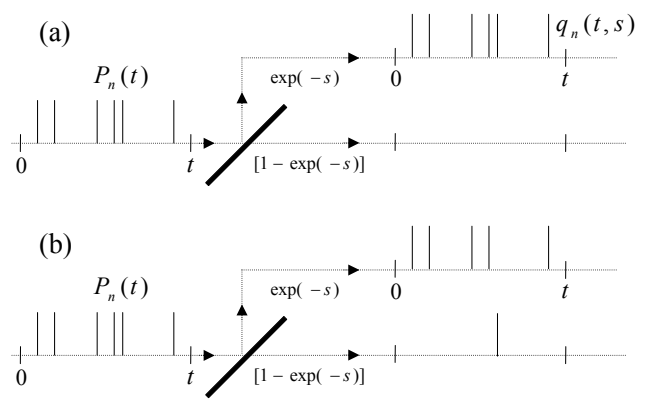

FIG. 1: Conditional counting scheme that leads to the realizations of $\left\{q_{n}(t, s)\right\}_{n=0}^{\infty}$. Each event of $\left\{P_{n}(t)\right\}_{n=0}^{\infty}$ (left vertical lines) is selected with probabilities $\exp [-s]$ and $(1-\exp [-s])$ in the upper and lower arms respectively. In (a) all events went in the upper arm, leading to a s-realization. (b) Realizations with at least one event in the lower arm are discarded.

ditional selection corresponds the factor $\exp [-s n]$ in the definition (17). Furthermore, $Z(t, s)$ can be read as the fraction of the total realizations of $\left\{P_{n}(t)\right\}_{n=0}^{\infty}$ that are taken as realizations of the set $\left\{q_{n}(t, s)\right\}_{n=0}^{\infty}$. Therefore, it provides the right normalization associated to the conditional selection. Alternatively, it can be read as the probability of not occurring any event in the lower arm in the interval $(0, t)$.

When $s<0$, it follows $\exp [-s]>1$. Therefore, the previous scheme does not apply. Nevertheless, a similar interpretation can be established. We realize that for any value of $s$ it is possible to invert Eq. (17) as

$$
P_{n}(t)=\frac{1}{Z_{q}(t,-s)} q_{n}(t, s) e^{s n}
$$

where we have defined the $q$-partition function

$$
Z_{q}(t, s) \equiv \sum_{n=0}^{\infty} q_{n}(t, s) e^{-s n}
$$

Notice that the normalization of the counting probabilities implies the relation $Z_{q}(t,-s)=1 / Z(t, s)$. For $s<0$, Eq. (19) has the same structure than Eq. (17). Hence, even in this case, the previous measurement scheme makes sense. Nevertheless, for $s<0$ the input signal must correspond to the realizations of $\left\{q_{n}(t, s)\right\}_{n=0}^{\infty}$ while the the output signal delivers the realizations defined by the set $\left\{P_{n}(t)\right\}_{n=0}^{\infty}$. Although this association is exact $(s<0)$, it does not provide a procedure for generating the $s$-ensemble from the original counting process. In fact, it solves the inverse problem.

The relation between the original ensemble and the $s$ ensemble can be extended to two arbitrary values, $s$ and $s^{\prime}$, of the pseudo chemical potential. We write

$$
q_{n}(t, s)=\frac{1}{Z\left(t, s, s^{\prime}\right)} q_{n}\left(t, s^{\prime}\right) e^{-\left(s-s^{\prime}\right) n},
$$

where the normalization here reads

$$
Z\left(t, s, s^{\prime}\right) \equiv \sum_{n=0}^{\infty} q_{n}\left(t, s^{\prime}\right) e^{-\left(s-s^{\prime}\right) n} .
$$

For $s>s^{\prime}$, the realizations of $\left\{q_{n}(t, s)\right\}_{n=0}^{\infty}$ follow from those of $\left\{q_{n}\left(t, s^{\prime}\right)\right\}_{n=0}^{\infty}$ by applying the conditional measurement procedure sketched in Fig. 1. The set $\left\{P_{n}(t)\right\}_{n=0}^{\infty}$ is associated to $s^{\prime}=0$. Furthermore, it is simple to realize why it is not possible to build up a measurement scheme for obtaining the realizations of $\left\{q_{n}\left(t, s^{\prime}\right)\right\}_{n=0}^{\infty}$ from those of $\left\{q_{n}(t, s)\right\}_{n=0}^{\infty}$, i.e., the case $s<s^{\prime}$. In fact, the realizations of the last counting process follow from a subset of the realizations of the former one, implying a lack of information which cannot be recovered from any measurement scheme.

From the definition of the conditional measurement scheme (Fig. 1) and the previous analysis, it is simple to realize that the thermodynamic response $\langle\langle N\rangle\rangle[$ Eq. [13)] is a strictly decreasing function of $s$, implying

$$
\lim _{s \rightarrow+\infty}\langle\langle N\rangle\rangle=0, \quad \lim _{s \rightarrow-\infty}\langle\langle N\rangle\rangle=\infty .
$$

These constraints imply the concavity of the free energy function $\Theta(s)$ and the limits

$$
\lim _{s \rightarrow+\infty} \Theta(s)=|C|, \quad \lim _{s \rightarrow-\infty} \Theta(s)=-\infty,
$$

where $C$ is a real constant. Consistently with the behavior of $\langle\langle N\rangle\rangle$, in general $\left\langle\left\langle\Delta N^{2}\right\rangle\right\rangle$ is neither an increasing nor a decreasing function of $s$.

The function $Z(t, s)$ measures the fraction of the number of realizations from the original counting process that are taken for the $s$-ensemble. The asymptotic behavior (7) implies that a reliable measurement of the set $\left\{q_{n}(t, s)\right\}_{n=0}^{\infty}$ [Eq. [17)] and the response functions involves a number of realizations of $\left\{P_{n}(t, s)\right\}_{n=0}^{\infty}$ that scales as $\exp \left[t^{*} \Theta(s)\right]$, where $t^{*}$ is larger than the transitory time interval. From a numerical point of view, this condition is very restrictive. In fact, it can take a long time before a realization is generated. In spite of this severe limitation, below (Fig. 3) we show that the measurement scheme can in fact be numerically implemented for moderate values of $s$, i.e., $s \approx 0$, such that the product $t^{*} \Theta(s)$ does not assume very high values.

\section{RENEWAL COUNTING PROCESSES}

While the thermodynamic potential $\Theta(s)$ provides a complete description of LD fluctuations, its functional form depends on each specific case. In contrast, some general results can be established from the statistical mechanics formulation based on the set $\left\{q_{n}(t, s)\right\}_{n=0}^{\infty}$. Here, we use this advantage for providing a general characterization of an arbitrary renewal counting process [2, 3] .

A renewal point process is defined by a WTD satisfying $w(t) \geq 0$ and the normalization $\int_{0}^{\infty} d t^{\prime} w\left(t^{\prime}\right)=1$. It defines the probability distribution of the (random) time interval between consecutive events. Therefore, the count- 
ing probabilities $\left\{P_{n}(t, s)\right\}_{n=0}^{\infty}$ can be written as 31 ]

$$
\begin{aligned}
& P_{0}(t)=1-\int_{0}^{t} d t^{\prime} w\left(t^{\prime}\right) \\
& P_{n}(t)=\int_{0}^{t} d t^{\prime} w\left(t-t^{\prime}\right) P_{n-1}\left(t^{\prime}\right) .
\end{aligned}
$$

By working in a Laplace domain, it is simple to derive the equivalent evolution equations

$$
\begin{aligned}
\frac{d}{d t} P_{0}(t) & =-\int_{0}^{t} d t^{\prime} K\left(t-t^{\prime}\right) P_{0}\left(t^{\prime}\right) \\
\frac{d}{d t} P_{n}(t) & =-\int_{0}^{t} d t^{\prime} K\left(t-t^{\prime}\right)\left[P_{n}\left(t^{\prime}\right)-P_{n-1}\left(t^{\prime}\right)\right]
\end{aligned}
$$

The memory kernel $K(t)$ is defined in the Laplace domain, $g(u)=\int_{0}^{\infty} d t g(t) \exp [-u t]$, as

$$
K(u)=\frac{w(u)}{P_{0}(u)}=\frac{u w(u)}{1-w(u)} .
$$

While the kernel $K(t)$ does not have a straightforward physical interpretation, its time integral does. After defining $f(t) \equiv \int_{0}^{t} d t^{\prime} K\left(t^{\prime}\right)$, or equivalently in the Laplace domain $f(u)=K(u) / u$, from Eq. (27) we get

$$
f(u)=\frac{w(u)}{1-w(u)}=\sum_{n=1}^{\infty}[w(u)]^{n} .
$$

By writing this expression in the time domain in terms of successive convolutions of $w(t)$, we realize that $f(t) d t$ provides the probability of observing an event in $(t, t+d t)$ independently of the occurrence of any extra events in previous time interval $(0, t)[31]$. In contrast, $w(t) d t$ has the same interpretation under the condition that not any event occurs in $(0, t)$.

\section{A. s-ensemble}

As the renewal property implies the absence of memory between consecutive events, the $s$-ensemble (17) can easily be characterized. From Eq. (26), the evolution of the partition function (8) reads

$$
\frac{d}{d t} Z(t, s)=-\left(1-e^{-s}\right) \int_{0}^{t} d t^{\prime} K\left(t-t^{\prime}\right) Z\left(t^{\prime}, s\right),
$$

whose solution in the Laplace domain is

$$
Z(u, s)=\frac{1}{u+K(u)\left(1-e^{-s}\right)} .
$$

By writing the survival probability in the Laplace domain as $P_{0}(u)=[u+K(u)]^{-1}$ [see Eq. (26)], it follows that $Z(u, s)$ with $s>0$ can be read as the Laplace transform of a survival probability defined with the normalized kernel $K(u) \rightarrow\left(1-e^{-s}\right) K(u)$, which in turn implies $f(u) \rightarrow\left(1-e^{-s}\right) f(u)$. Therefore, we confirm that in fact $Z(t, s)$ can be identified with the survival probability of the lower arm of the measurement scheme of Fig. 1.

From its definition and using the renewal property, Eq. (25), $Z(t, s)$ can alternatively be written as

$$
Z(t, s)=P_{0}(t)+\int_{0}^{t} d t^{\prime} w\left(t-t^{\prime}\right) e^{-s} Z\left(t^{\prime}, s\right) .
$$

Then, as $q_{0}(t, s)=P_{0}(t) / Z(t, s)$ it follows

$$
q_{0}(t, s)=1-\int_{0}^{t} d t^{\prime} w_{q}\left(t, t^{\prime}, s\right) .
$$

Furthermore, from Eqs. (17) and (25), for $n \geq 1$ we get

$$
q_{n}(t, s)=\int_{0}^{t} d t^{\prime} w_{q}\left(t, t^{\prime}, s\right) q_{n-1}\left(t^{\prime}, s\right)
$$

where we have defined

$$
w_{q}\left(t, t^{\prime}, s\right) \equiv e^{-s} w\left(t-t^{\prime}\right) \frac{Z\left(t^{\prime}, s\right)}{Z(t, s)} .
$$

This expression, together with relations (32) and (33), imply that the $s$-ensemble is a non-stationary renewal counting process [compare with Eqs. (25)]. In fact, here the WTD $w_{q}\left(t, t^{\prime}, s\right)$ not only depends on the interval between successive events, but also on the time of the last event. Consistently with the measurement scheme of Fig. 1, this dependence is introduced by the partition function factors. By introducing the interval $\tau=t-t^{\prime}$ between two consecutive events, we write $w_{q}(t, t-\tau, s)=e^{-s} w(\tau) Z(t-\tau, s) / Z(t, s)$. In this way, $w_{q}(t, t-\tau, s)$ can be read as the probability density for observing an event at $t$ given that the last one occurred at time $(t-\tau)$. The normalization $\lim _{t \rightarrow \infty} \int_{0}^{t} d \tau w_{q}(t, t-\tau, s)=1$, follows from Eqs. (31) and (32) after using that $\lim _{t \rightarrow \infty} q_{0}(t, s)=0$. In the long time regime, where the partition function can be approximated by an exponential decay, Eq. (17), we get

$$
\begin{aligned}
w_{q}^{\infty}(\tau, s) & \equiv \lim _{t \rightarrow \infty} w_{q}(t, t-\tau, s) \\
& =e^{-s} w(\tau) \exp [\tau \Theta(s)]
\end{aligned}
$$

Therefore, in the regime where the LD approach applies, the counting process defined by the set $\left\{q_{n}(t, s)\right\}_{n=0}^{\infty}$ becomes a stationary renewal one, which in turn is controlled by the WTD $w_{q}^{\infty}(\tau, s)$. This is one of the central results of this section. Notice that it is valid for any value of $s$. On the other hand, one can also associate to the $s$-ensemble a non-conditional probability distribution

$$
f_{q}\left(t, t^{\prime}, s\right)=w_{q}\left(t, t^{\prime}, s\right)+\int_{t^{\prime}}^{t} d t_{1} w_{q}\left(t, t_{1}, s\right) f_{q}\left(t_{1}, t^{\prime}, s\right)
$$

which defines the probability density of observing an event in $(t, t+d t)$ given that one occurred at $t^{\prime}$ and independently of the occurrence of any extra events in the 
time interval $\left(t, t^{\prime}\right)$. By solving Eq. (36) iteratively, after some calculations we get

$$
f_{q}\left(t, t^{\prime}, s\right)=e^{-s} \digamma\left(t-t^{\prime}, s\right) \frac{Z\left(t^{\prime}, s\right)}{Z(t, s)},
$$

where $\digamma(t, s)$ is defined in the Laplace domain as

$$
\digamma(u, s)=K(u) Z(u, s)=\frac{K(u)}{u+K(u)\left(1-e^{-s}\right)} .
$$

Consistently, $f_{q}\left(t, t^{\prime}, 0\right)=f\left(t-t^{\prime}\right)$. Furthermore, in correspondence with Eq. (35), we define $f_{q}^{\infty}(\tau, s)=$ $\lim _{t \rightarrow \infty} f_{q}(t, t-\tau, s)$, which reads

$$
f_{q}^{\infty}(\tau, s)=e^{-s} \digamma(\tau, s) \exp [\tau \Theta(s)] .
$$

From its definition [31], it follows the relation $\lim _{\tau \rightarrow \infty} f_{q}^{\infty}(\tau, s)=\langle\langle N\rangle\rangle$, Eq. (13).

\section{B. Free energy and response functions}

The underlying renewal property of the $s$-ensemble allows us to get some general expressions for the free energy and its response functions. The Laplace transform of $w_{q}^{\infty}(\tau, s)$ [Eq. [35)] with respect to $\tau$ can be written as $w_{q}^{\infty}(u, s)=e^{-s} w(u-\Theta(s))$. As this function satisfies the normalization $\int_{0}^{\infty} d \tau w_{q}^{\infty}(\tau, s)=1$, or equivalently $\left.w_{q}^{\infty}(u, s)\right|_{u=0}=1$, we obtain

$$
\left.w(u-\Theta(s))\right|_{u=0}=e^{s} .
$$

By using this relation and the asymptotic values (24) one can deduce the relation

$$
\lim _{s \rightarrow-\infty} \Theta(s) \approx \gamma_{0}\left(1-e^{-\alpha_{0} s}\right),
$$

valid when $w(t)$ has a characteristic short time scale, i.e., $\lim _{u \rightarrow \infty} w(u) \simeq\left(\gamma_{0} / u\right)^{1 / \alpha_{0}}$.

The Laplace transform of $w(t)$ also provides an equation for getting the free energy function. In fact, it is the smaller solution of Eq. (40). This relation is valid for any (ergodic) renewal process. Notice that the same kind of relations follow from definition (77). In fact, by using the residues theorem, $\Theta(s)$ can be determined as the smaller root of the denominator of Eq. (30).

As the process defined by the set $\left\{q_{n}(t, s)\right\}_{n=0}^{\infty}$ is a renewal one in the stationary regime, the thermodynamic response functions [Eq. (18)] can be derived from $w_{q}^{\infty}(\tau, s)$. In fact, by defining the time interval moments

$$
\tau_{n}(s) \equiv \int_{0}^{\infty} w_{q}^{\infty}(\tau, s) \tau^{n} d \tau,
$$

and by using the renewal property, it follows

$$
\langle\langle N\rangle\rangle=\frac{1}{\tau_{1}(s)}, \quad\left\langle\left\langle\Delta N^{2}\right\rangle\right\rangle=\frac{\tau_{2}(s)-\left[\tau_{1}(s)\right]^{2}}{\left[\tau_{1}(s)\right]^{3}},
$$

where we have used that $\langle\langle N\rangle\rangle$ and $\left\langle\left\langle\Delta N^{2}\right\rangle\right\rangle$ measure the linear growth of the first two cumulants of the counting process defined by the set $\left\{q_{n}(t, s)\right\}_{n=0}^{\infty}$ (see page 496 of Ref. 31]). Similar relations are valid for higher response functions, i.e., $w_{q}^{\infty}(\tau, s)$ completely determine the thermodynamic formalism.

\section{Applications}

Below, we show some applications that rely on the previously developed results.

\section{Scale invariant renewal process}

In Ref. 28, 29], a scale invariant property was found in a class of photon counting process. It is defined by the condition

$$
\frac{\left\langle\left\langle\Delta N^{2}\right\rangle\right\rangle}{\langle\langle N\rangle\rangle}=\alpha,
$$

where the response functions follow from Eq. (18), and $\alpha$ is a positive real constant, $0<\alpha<\infty$. Hence, the normalized fluctuations do not depend on $s$. The counting processes $\left\{P_{n}(t)\right\}_{n=0}^{\infty}$ and $\left\{q_{n}(t, s)\right\}_{n=0}^{\infty}$, for any value of $s$, are then sub-Poissonian for $0<\alpha<1$, while for $1<\alpha<\infty$, becomes super-Poissonian [2-4]. Here, we demonstrate that the scale invariant property is always satisfied by a class of (gamma) renewal process [2-4], which are defined by the WTD

$$
w(u)=\left(\frac{\gamma}{u+\gamma}\right)^{1 / \alpha} .
$$

In the time domain it reads

$$
w(t)=\frac{1}{\Gamma(1 / \alpha)}(\gamma t)^{\frac{1}{\alpha}-1} \gamma e^{-\gamma t} .
$$

The average interval between consecutive events is given by $\int_{0}^{\infty} w(t) t d t=(\alpha \gamma)^{-1}$. By working the expressions (25) in the Laplace domain, the counting probabilities read

$$
P_{n}(t)=\frac{\Gamma\left(\frac{n+1}{\alpha}, \gamma t\right)}{\Gamma\left(\frac{n+1}{\alpha}\right)}-\frac{\Gamma\left[\frac{n}{\alpha}, \gamma t\right]}{\Gamma\left(\frac{n}{\alpha}\right)}\left(1-\delta_{n 0}\right),
$$

where $\Gamma[k, x]$ is the incomplete gamma function $\Gamma[k, x] \equiv$ $\int_{x}^{\infty} z^{k-1} e^{-z} d z$, and $\Gamma(k)$ is the Euler gamma function, i.e., $\Gamma(k)=\Gamma[k, 0]$. In the long time regime, $\gamma t \gg 1$, it is valid the approximation

$$
P_{n}(t) \simeq \frac{1}{\alpha \Gamma\left(\frac{n+1}{\alpha}\right)}(\gamma t)^{\frac{n+1}{\alpha}-1} \exp [-\gamma t] .
$$

From this expression, it follows the LD function [Eq. (44)]

$$
\varphi(n)=\gamma\left\{1-\frac{n}{\alpha \gamma}\left[1-\log \left(\frac{n}{\alpha \gamma}\right)\right]\right\},
$$


$(n / t) \rightarrow n$. Its Legendre-Fenchel transformation [Eq. (6)] leads to the grand potential

$$
\Theta(s)=\gamma\left(1-e^{-\alpha s}\right) .
$$

Consistently, this result straightforwardly follows from Eq. (40). Alternatively, Eq. (30) leads to the partition function

$$
Z(u, s)=\frac{1}{u} \frac{(u+\gamma)^{1 / \alpha}-\gamma^{1 / \alpha}}{(u+\gamma)^{1 / \alpha}-\gamma^{1 / \alpha} e^{-s}} .
$$

From the residues theorem, the free energy (50) is recovered. In fact, $u=-\Theta(s)$ cancels the denominator of this expression. The grand potential (50) implies the thermodynamic response functions [Eq. (18)]

$$
\langle\langle N\rangle\rangle=\alpha \gamma e^{-\alpha s}, \quad\left\langle\left\langle\Delta N^{2}\right\rangle\right\rangle=\alpha^{2} \gamma e^{-\alpha s} .
$$

Trivially, these expressions satisfy the invariant property Eq. (44).

While it is not possible to find a simple expression for the non-stationary WTD $w_{q}\left(t, t^{\prime}, s\right)$ [Eq. (34)], the stationary one [Eq. (35)] reads

$$
w_{q}^{\infty}(\tau, s)=\frac{1}{\Gamma(1 / \alpha)}\left(\gamma_{s} \tau\right)^{\frac{1}{\alpha}-1} \gamma_{s} \exp \left[-\gamma_{s} \tau\right]
$$

where the renormalized rate reads

$$
\gamma_{s} \equiv \gamma \exp [-\alpha s]
$$

The stationary $\operatorname{WTD} w_{q}^{\infty}(\tau, s)$ is the same as the original one [see Eq. (46)] under the replacement $\gamma \rightarrow$ $\gamma e^{-\alpha s}$. Thus, the counting process defined by the set $\left\{q_{n}(t, s)\right\}_{n=0}^{\infty}$, in the asymptotic regime, can be obtained from the original one after a time rescaling

$$
\lim _{t \rightarrow \infty} q_{n}(t, s) \simeq \lim _{t \rightarrow \infty} P_{n}\left(t e^{-\alpha s}\right) .
$$

This condition was derived from the WTD Eq. (45). Nevertheless, it is simple to prove that its fulfilment is sufficient to guaranty the invariance property (44). By using that $Z_{q}(t,-s)=1 / Z(t, s)$ [see Eq. (20)], from (55) it follows the equivalent condition

$$
\Theta(-s)=-\Theta(s) e^{\alpha s}
$$

which in turn allows to write an equation for $(d / d s) \Theta(s)$. Under the constraints (56), continuous derivatives in $s=$ 0 , and $\Theta(0)=0$, it has a unique non-null solution that is given by Eq. (50). Notice that this derivation does not rely on the renewal property. Nevertheless, the previous one guarantees that $\left\{P_{n}(t)\right\}_{n=0}^{\infty}$ asymptotically converges to a renewal process defined by the WTD (45).

When $\alpha=1$, the WTD (45) becomes an exponential one, $w(t)=\gamma \exp (-\gamma t)$. Thus, $f(t)=\gamma \theta(t)$ [Eq. (28)] where $\theta(t)$ is the step function, implying a local in time evolution $[K(t)=\gamma \delta(t)]$ of the counting probabilities [Eq. (26) ]. Their solution read $P_{n}(t)=(\gamma t)^{n} \exp [-\gamma t] / n$ !, i.e., the very well-known Poisson process [1 4$]$. The partition function [Eq. (8)] reads

$$
Z(t, s)=\exp \left[-\gamma t\left(1-e^{-s}\right)\right], \quad \alpha=1,
$$

which in turn implies the free energy (50) and its associated response functions with $\alpha=1$. The $s$-ensemble [Eq. (17)] at "any particular time" can easily be written as $q_{n}(t, s)=\left(e^{-s} \gamma t\right)^{n} \exp \left[-e^{-s} \gamma t\right] / n$ !. Consistently, these probabilities also correspond to a Poisson process with the normalized rate $\gamma \rightarrow e^{-s} \gamma$. Its associated nonstationary WTD [Eq. (34)] is stationary at all times

$$
w_{q}\left(t, t^{\prime}, s\right)=\left(\gamma e^{-s}\right) \exp \left[-\left(\gamma e^{-s}\right)\left(t-t^{\prime}\right)\right], \quad \alpha=1,
$$

i.e., at any particular time it only depends on the difference $\left(t-t^{\prime}\right)$. This property is only valid for the Poisson process, $\alpha=1$.

Scale invariant photon-emission process. In Ref. [28] it was analyzed the case of a two-level fluorescent system, where an external laser field induces a continuous emission of photons at random times. The photon counting process is a renewal one, being defined by the WTD

$$
w(u)=\frac{\gamma / 2}{u+\gamma / 2} \frac{\Omega^{2}}{u^{2}+u \gamma+\Omega^{2}} .
$$

Here, $u$ is the Laplace variable, $\gamma$ denotes the natural decay rate of the system while the Rabi frequency $\Omega$ measures the system-laser coupling. The average time is $\int_{0}^{\infty} w(t) t d t=\left[\gamma \Omega^{2} /\left(\gamma^{2}+2 \Omega^{2}\right)\right]^{-1}$. When $2 \Omega=\gamma$, it was found the invariant scale property (44) with $\left\langle\left\langle\Delta N^{2}\right\rangle\right\rangle /\langle\langle N\rangle\rangle=1 / 3$. In this situation, (59) becomes

$$
w(u)=\left(\frac{\Omega}{u+\Omega}\right)^{3},
$$

which in the time domain leads to $w(t)=$ $\frac{1}{2} \Omega^{3} t^{2} \exp [-\Omega t]$. These expressions and the previous results confirm that in fact $\alpha=1 / 3$ [see Eq. (45)], showing the consistence of the present approach.

\section{Shift closure property}

With $\{\xi\}$ we denote the characteristic parameters (rates, etc.) that determine a given counting process $\left\{P_{n}(t)\right\}_{n=0}^{\infty}$. Evidently, its associated free energy depends on $\{\xi\}$, property denoted as $[\Theta(s)]_{\{\xi\}}$. We define a shift closure property by the condition

$$
[\Theta(s)]_{\left\{\xi\left(s_{0}\right)\right\}}=\left[\Theta\left(s+s_{0}\right)-\Theta\left(s_{0}\right)\right]_{\{\xi\}},
$$

where $\left\{\xi\left(s_{0}\right)\right\}$ are new characteristic parameters that depend on $s_{0}$. Note that $\left[\Theta\left(s+s_{0}\right)-\Theta\left(s_{0}\right)\right]_{\{\xi\}}$ is the free energy function of the counting process $\left\{q_{n}\left(t, s_{0}\right)\right\}_{n=0}^{\infty}$. In fact, all response functions, i.e., its derivatives with respect to $s$ [Eq. (18)], are the same as those of $\Theta(s)$ shifted by $-s_{0}$. Hence, the condition (61) tells us that $\left\{q_{n}\left(t, s_{0}\right)\right\}_{n=0}^{\infty}$ (the $s_{0}$-ensemble) belongs to the family of 
processes obtained from $\left\{P_{n}(t)\right\}_{n=0}^{\infty}$ by a change of characteristic parameters, $\{\xi\} \rightarrow\left\{\xi\left(s_{0}\right)\right\}$. Consequently, the fulfilment of Eq. (61) allows us to generate the realizations of the $s$-ensemble by changing the parameters of the original counting process. Equivalently, it implies that the change of parameters $\{\xi\} \rightarrow\left\{\xi\left(s_{0}\right)\right\}$ in the definition of $\left\{P_{n}(t)\right\}_{n=0}^{\infty}$ only produce a shifting of all of its response functions.

Scale invariant processes, Eq. (50), satisfy the shift closure condition with the mapping $\{\gamma\} \rightarrow\left\{\gamma e^{-\alpha s_{0}}\right\}$ [Eq. (54)]. In general, it is very difficult to check the validity of (61). Nevertheless, for renewal processes it can alternatively be written in terms of the stationary WTD (35) as

$$
w_{q}^{\infty}(\tau, s)=[w(\tau)]_{\{\xi(s)\}} .
$$

Hence, $w_{q}^{\infty}(\tau, s)$ follows from $w(\tau)$ after replacing $\{\xi\} \rightarrow$ $\{\xi(s)\}$. When satisfied, this equation is not only equivalent to (61) but also provides a simple way for obtaining the parameters mapping $\{\xi\} \rightarrow\left\{\xi\left(s=s_{0}\right)\right\}$.

A broad class of WTDs satisfy the shift closure property. For example, consider in the Laplace domain $w(u)=\prod_{i} \lambda_{i} /\left(u+\lambda_{i}\right)$. By using the normalization condition (40), we get

$$
w_{q}^{\infty}(u, s)=\prod_{i} \frac{\lambda_{i}(s)}{u+\lambda_{i}(s)}, \quad \lambda_{i}(s)=\lambda_{i}-\Theta(s) .
$$

Notice that $w_{q}^{\infty}(u, s)$ have the same structure than $w(u)$ with the rescaled parameters $\{\xi(s)\}=\left\{\lambda_{i}(s)\right\}$. This simple result guarantees the validity of Eq. (61). In general, $\lambda_{i}$ may be complex numbers $\left(\operatorname{Re}\left[\lambda_{i}\right]>0\right)$, which in turn are functions of experimental parameters. Eq. (59) has this structure, with $\lambda_{i}=\lambda_{i}\left(\gamma, \Omega^{2}\right)$. In this case, we checked that $w_{q}^{\infty}(u, s)$ can be written as $w(u)$ after replacing $\gamma \rightarrow \gamma(s)$ and $\Omega^{2} \rightarrow \Omega^{2}(s)$, where $\gamma(s)=$ $\gamma-2 \Theta(s)$, and $\Omega^{2}(s)=\Omega^{2}+\Theta(s)[\Theta(s)-\gamma]$, where $\Theta(s)$ is defined by Eq. (35) of Ref. 29]. Notice that the parameter transformation satisfies $\gamma^{2}(s)-4 \Omega^{2}(s)=\gamma^{2}-4 \Omega^{2}$.

Another class of WTDs that satisfy the shifting condition (62) is given by $w(t)=\sum_{k} p_{k} \gamma_{k} e^{-\gamma_{k} t}$, with $\sum_{k} p_{k}=$ $1,0<p_{k}<1$. Thus, in this case $\{\xi\}=\left\{\gamma_{k}, p_{k}\right\}$. From Eq. (35), we get

$$
w_{q}^{\infty}(\tau, s)=\sum_{k} p_{k}(s) \gamma_{k}(s) e^{-\gamma_{k}(s) \tau},
$$

where the parameters $\{\xi(s)\}=\left\{\gamma_{k}(s), p_{k}(s)\right\}$ are

$$
\gamma_{k}(s)=\gamma_{k}-\Theta(s), \quad p_{k}(s)=p_{k} \frac{e^{-s} \gamma_{k}}{\gamma_{k}-\Theta(s)},
$$

with $\gamma_{k}(s)>0$ and $\sum_{k} p_{k}(s)=1$. Notice that $w_{q}^{\infty}(\tau, s)$ can also be written as a superposition of Poisson distributions, which guarantees the fulfilment of (61) with the mapping $\left\{\gamma_{k}, p_{k}\right\} \rightarrow\left\{\gamma_{k}\left(s_{0}\right), p_{k}\left(s_{0}\right)\right\}$, Eq. (65).

In order to exemplify the previous results, we consider a bi-exponential WTD

$$
w(t)=(1-p) \gamma_{>} e^{-\gamma>t}+p \gamma_{<} e^{-\gamma<t},
$$

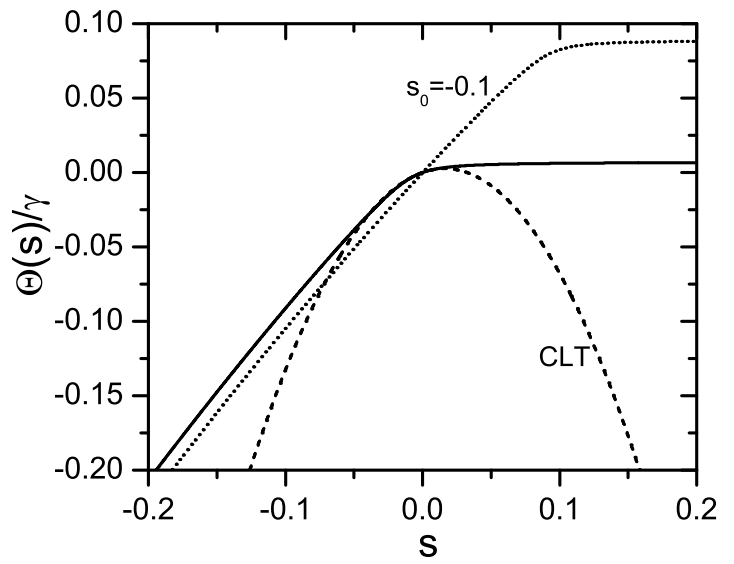

FIG. 2: Free energy function (69) (full line) in terms of $s$. The parameters of the WTD [Eq. [66)] are $\gamma_{</} / \gamma_{>}=0.007$ and $p=0.015\left[\gamma / \gamma_{>}=0.9815\right]$. The dotted line corresponds to Eq. 61 with $s_{0}=-0.1$. The short-dashed line is the estimation based on the CLT, Eq. (10).

where $\gamma_{>}$and $\gamma_{<}$are the characteristic rates and the parameter $p$ satisfies $0 \leq p \leq 1$. The kernel (27) defines the evolution of the counting probabilities $\left\{P_{n}(t)\right\}_{n=0}^{\infty}$ [Eq. (26)]. It reads

$$
K(t)=\gamma\{\delta(t)-\beta \exp [-\eta t]\},
$$

where the characteristic parameters are

$$
\begin{aligned}
\gamma & \equiv(1-p) \gamma_{>}+p \gamma_{<}, \\
\beta & \equiv\left\{(1-p) \gamma_{>}^{2}+p \gamma_{<}^{2}-\gamma^{2}\right\} / \gamma, \\
\eta & \equiv(1-p) \gamma_{<}+p \gamma_{>} .
\end{aligned}
$$

The grand potential [Eq. (15)] follows from the relation (40). Equivalently, by using the residues theorem it can be obtained from the smaller root of the denominator of Eq. (30), $u^{2}+u\left[\gamma\left(1-e^{-s}\right)+\eta\right]+(\eta-\beta) \gamma\left(1-e^{-s}\right)=0$. We get

$$
\begin{aligned}
\Theta(s)= & \frac{\gamma}{2}\left(1-e^{-s}\right)+\frac{\eta}{2}-\frac{1}{2}\left\{\left[\eta-\gamma\left(1-e^{-s}\right)\right]^{2}\right. \\
& \left.+4 \beta \gamma\left(1-e^{-s}\right)\right\}^{1 / 2} .
\end{aligned}
$$

In Fig. 2 we plot $\Theta(s)$ (full line) as a function of the chemical potential $s$ for a particular set of parameter values of the WTD (66). The limits (24) are satisfied. The dotted line corresponds to $\left[\Theta\left(s+s_{0}\right)-\Theta\left(s_{0}\right)\right]$, with $s_{0}=-0.1$. Consistently, we checked that the same (shifted) free energy function follows from Eq. (69) under parameter replacements $\{\xi\}=\left\{p, \gamma_{>}, \gamma_{<}\right\} \rightarrow$ $\left\{p\left(s_{0}\right), \gamma_{>}\left(s_{0}\right), \gamma_{<}\left(s_{0}\right)\right\}=\left\{\xi\left(s_{0}\right)\right\}$, defined by Eq. (65). This example explicitly shows the meaning of the shift closure condition.

In Fig. 3 we plot the response functions, Eq. (18). Consistently, they fulfill Eq. (43). We also checked that the shifted response functions coincide with the 

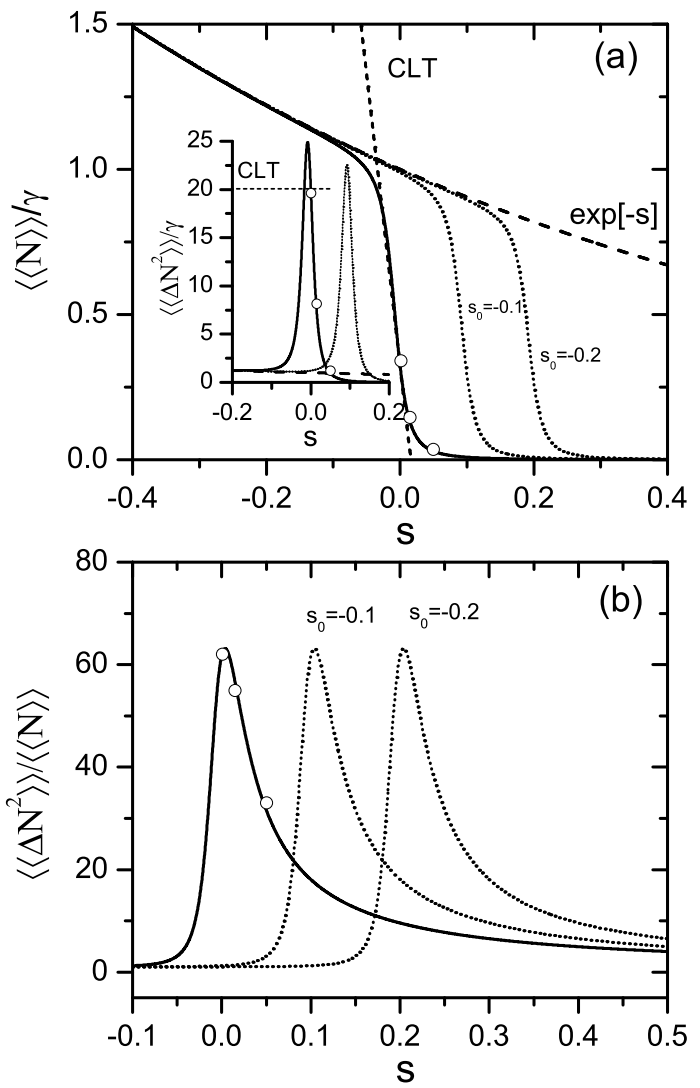

FIG. 3: (a) First and second (inset) response functions, Eq. (18). The parameters of the WTD are the same than in Fig. 2. The dotted lines correspond to a set of different parameters values obtained from Eq. (65) with $s_{0}=-0.1$ and $s_{0}=-0.2$. The circles correspond to the numerical values obtained from the measurement scheme of Fig. 1 with the transformations $s=0 \rightarrow s=0.001,0.015,0.05$. The short-dashed lines are the estimations based on the CLT, Eq. (10). The long-dashed lines correspond to the response functions of a Poisson process with rate $\gamma$ (see text). (b) Normalized second response function $\left\langle\left\langle\Delta N^{2}\right\rangle\right\rangle /\langle\langle N\rangle\rangle$.

derivative with respect to $s$ of $[\Theta(s)]_{\left\{\xi\left(s_{0}\right)\right\}}$. In Fig. 3(a) all plotted functions are normalized by the rate $\gamma$, Eq. (68), which is different for each set of parameter values, $s=0,-0.1,-0.2$. Hence, the shifted response functions differ from the original one $(s=0)$ in a multiplicative factor. This effect is absent in the normalized second response function $\left\langle\left\langle\Delta N^{2}\right\rangle\right\rangle /\langle\langle N\rangle\rangle$, Fig. 3(b).

\section{Intermittent renewal process and finite-size effects}

In order to enlighten the LD method, in Figs. 2 and 3 we show the predictions corresponding to the CLT (short-dashed lines), i.e., $\Theta(s) \stackrel{c l t}{=} s\left(\left.\langle\langle N\rangle\rangle\right|_{s=0}\right)-$ $\left(s^{2} / 2\right)\left(\left.\left\langle\left\langle\Delta N^{2}\right\rangle\right\rangle\right|_{s=0}\right)$, Eq. (10). Thus, the first response function has a linear dependence on $s$ and the second one becomes a constant. While the first approximation is valid in a small region around $s \approx 0$, the second one
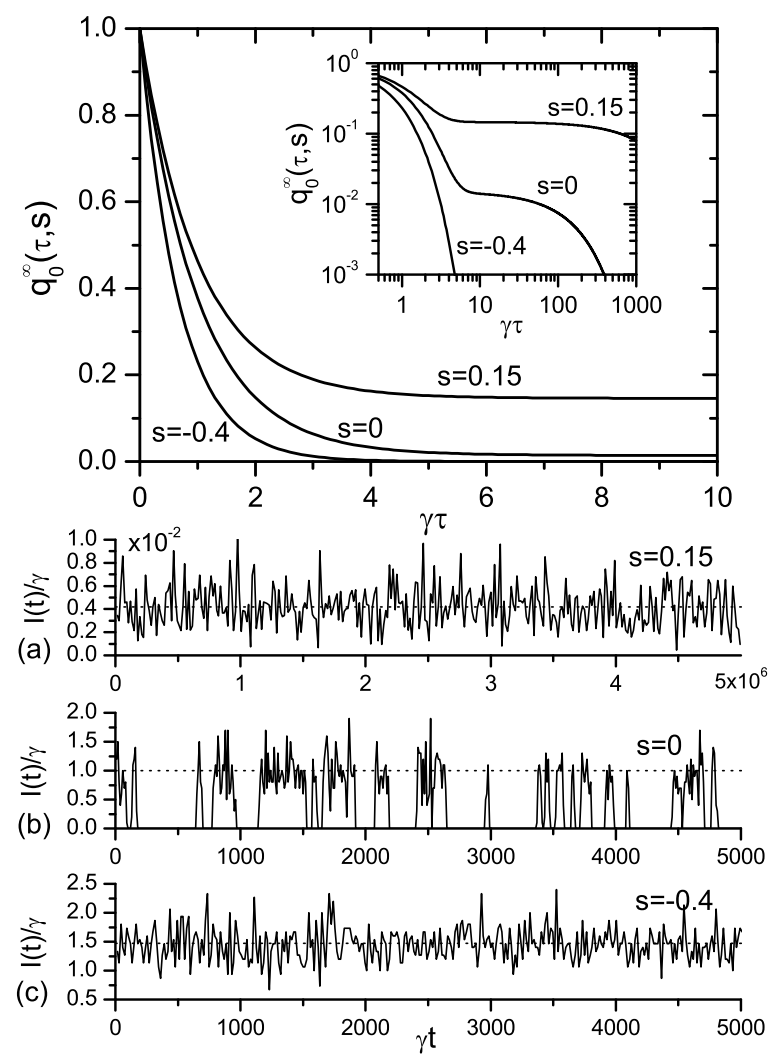

FIG. 4: Stationary survival probability (70) as a function of time for different values of $s$. The inset corresponds to a loglog plot. The intensity realizations (see text) ploted in (a), (b), and (c) correspond to $s=+0.15, s=0$, and $s=-0.4$ respectively. The parameters of the WTD (66) are the same than in Fig. 2.

only applies at $s=0$.

The previous analysis implies that the CLT approximation could miss a phase transition within the thermodynamic approach. In contrast, the response functions plotted in Fig. 3 develops properties consistent with finite-size effects in a first order transition [32, 33]. In fact, the first response function changes abruptly around $s \approx 0$, while the second one develops a narrow peak. As shown in Refs. 28, 29] these features can be related to an intermittence (blinking) property of the counting process. Here, we show that renewal processes, independently of the underlying dynamics, may also develop similar phenomena.

The main features of the response functions shown in Fig. 3 can be easily interpreted in terms of the $s$-ensemble dynamics, which in turn is defined by the stationary WTD (35). In Fig. 4, in order to show the $s$-dependence of $w_{q}^{\infty}(\tau, s)$, we plot its associated survival probability

$$
q_{0}^{\infty}(\tau, s) \equiv 1-\int_{0}^{\tau} d \tau^{\prime} w_{q}^{\infty}\left(\tau^{\prime}, s\right),
$$

or equivalently $w_{q}^{\infty}(\tau, s)=-(d / d \tau) q_{0}^{\infty}(\tau, s)$. This function allows to generate the ensemble of realizations con- 
sistent with the asymptotic behavior of the counting probabilities $\left\{q_{n}(t, s)\right\}_{n=0}^{\infty}$. In fact, the time interval between consecutive events can be determined by solving the equation $q_{0}^{\infty}(\tau, s)=r$, where $r$ is a random number in the interval $(0,1)$.

The previous algorithm allows us to check the measurement scheme defined in Fig. 1. We numerically implemented the conditional selection to the generated realizations $(s=0)$ and calculated numerically $\langle\langle N\rangle\rangle$ from the asymptotic behavior (13). $\left\langle\left\langle\Delta N^{2}\right\rangle\right\rangle$ can be obtained in a similar way as the growing rate of the quadratic fluctuations. In Fig. 3 we show the results (circles) for the transformations $s=0 \rightarrow s=0.001,0.015,0.05$. Even for values of $s$ where the predictions of the CLT do not apply, the numerical estimations agree with the theoretical results.

In Fig. 4, for each chosen value of $s$, we also plotted the intensity $I(t)$ associated to an arbitrary stochastic realization of the $s$-ensemble. They were obtained numerically from $q_{0}^{\infty}(\tau, s)$. The intensity is defined as $I(t)=(d / d t) n_{s t}^{(s)}(t) \approx\left[n_{s t}^{(s)}(t+\Delta)-n_{s t}^{(s)}(t)\right] / \Delta$, where $n_{s t}^{(s)}(t)$ is the stochastic number of events up to time $t$ and $\Delta$ define an adequate discretization time. Consistently, one can always find a $\Delta$ where $I(t)$ fluctuates around $\langle\langle N\rangle\rangle$ (Fig. 4(a) and (c), horizontal dotted lines). Nevertheless, for $s=0$, i.e., for the counting probabilities $\left\{P_{n}(t)\right\}_{n=0}^{\infty}$, one can also find a discretization time where the intensity develops an intermittence phenomenon, i.e., there exist successive time intervals where either many events happen or none happen at all.

In the thermodynamic picture, the active and inactive periods are associated to two different phases, where finite-size effects lead to the transitions between them [29]. In the present approach, the intermittence phenomenon can be related to the WTD $w(t)$ [Eq. (66)]. For the chosen parameter values, which satisfy $p \ll 1$ and $\gamma_{<} / \gamma_{>} \ll 1, w(t)$ develops two different time scales, where the weight of one of them is much higher than the other one. This feature is evident in Fig. $4(s=0)$. Hence, the origin of the intermittence property follows straightforwardly from the previous defined algorithm [11]. The active and inactive time periods arise respectively from the short and long time behaviors of the WTD. Consistently, the intensity of the active periods fluctuates around $\gamma_{>} \approx \gamma$ (dotted line in Fig. 4b).

The intensity realizations show that when $s$ departs from zero, the intermittence phenomenon is lost. In the thermodynamic picture this property means that one of the two phases becomes dominant or stable. Specifically, the active and inactive phases are dominant for $s<0$ and $s>0$ respectively [see Fig. 3(a)]. Here, these conclusions can be related to the structure of the stationary WTD $w_{q}^{\infty}(\tau, s)[$ Eq. (35)].

The free energy $\Theta(s)$ goes to higher negative values for increasing negative values of $s$ [see Fig. 2]. Hence, for $s<0$ the two time scales property is lost, and the exponential term $\exp [-\tau|\Theta(s)|]$ becomes dominant in Eq. (35). In consequence the $s$-ensemble, by using Eq. (41), can be approximated by a Poisson process $w_{q}^{\infty}(\tau, s) \approx$ $\gamma \exp [-\gamma \tau]$, i.e., the active phase. The Poisson property explains why for $s<0$ all (normalized) response functions, independently of $s_{0}$, converge to the same asymptotic curve [Fig. 3(a)], $\langle\langle N\rangle\rangle / \gamma \approx\left\langle\left\langle\Delta N^{2}\right\rangle\right\rangle / \gamma \approx \exp [-s]$.

For increasing values of $s>0$ the free energy is positive, inducing a slower decay of $w_{q}^{\infty}(\tau, s)$. As can be seen in Fig. 4, the survival probability maintains two different time scales, nevertheless their relative weights are similar. Therefore, in this case the intermittence phenomenon is also lost. Furthermore, as the weight of the larger time scale increases with $s$, the intensity is diminished, i.e., the inactive phase becomes dominant for increasing $s$.

From the previous analysis, we conclude that the abrupt changes of $\langle\langle N\rangle\rangle$ and $\left\langle\left\langle\Delta N^{2}\right\rangle\right\rangle$ around $s \approx 0$ reflect both the loss of the intermittence property for $|s|>0$, and the transition between the active and inactive phases. In the thermodynamic approach, the behavior around $s \approx 0$ is read and estimated from a thermodynamic-finite-size analysis [29]. Those results can be straightforwardly applied in the present context after decomposing the partition function, Eq. (29), as $Z(t, s)=Z_{A}(t)+Z_{I}(t)$, where the evolution of each contribution (Active, Inactive) can be written as

$$
\begin{aligned}
\frac{d Z_{A}(t, s)}{d t}= & -\gamma\left(1-e^{-s}\right) Z_{A}(t, s) \\
& -\Gamma_{A} Z_{A}(t, s)+\Gamma_{I} Z_{I}(t, s), \\
\frac{d Z_{I}(t, s)}{d t}= & +\Gamma_{A} Z_{A}(t, s)-\Gamma_{I} Z_{I}(t, s) .
\end{aligned}
$$

Here, $\Gamma_{A}=\beta, \Gamma_{I}=\eta-\beta=\gamma_{>} \gamma_{<} / \gamma$, and the initial conditions must to be $Z_{A}(0, s)=1, Z_{I}(0, s)=0$. This splitting allows to read the counting process $\left\{P_{n}(t)\right\}_{n=0}^{\infty}$ as a stochastic modulated Poissonian one [see Eq. (57)], whose rate $\gamma_{s t}(t)$ at random times adopts the values $\gamma_{A}=\gamma$ and $\gamma_{I}=0$. The probabilities of assuming each value are governed by a classical master equation with transition rates $\Gamma_{A}$ and $\Gamma_{I}$.

In a slow modulation limit [29], i.e., when $\Gamma_{A} / \gamma \ll 1$ and $\Gamma_{I} / \gamma \ll 1$, Eq. (71) leads to an intermittence phenomenon. In fact, these conditions guarantee the existence of periods of time with many consecutive events and periods where no events happen at all. The previous inequalities can be satisfied by demanding $p \ll 1$ and $\gamma_{<} \ll \gamma_{>}$, such that $\gamma_{<} / \gamma_{>} \approx \mathrm{O}(p)$. Hence, from Eq. (68) it follows $\Gamma_{A} \approx p \gamma_{<}$, and $\Gamma_{I} \approx \gamma_{<}$. The parameters chosen in Fig. 2 satisfy these constraints. On the other hand, from Ref. [29] we know that the transition rates control the thermodynamic finite-size effects. In fact, the wide $\sigma_{p}$ of the peak of the second response function $\left\langle\left\langle\Delta N^{2}\right\rangle\right\rangle$ [inset of Fig. 3(a)] here can be estimated as $\sigma_{p} \approx 2\left(\Gamma_{A}+\Gamma_{I}\right) / \gamma \approx 2 p$ (see Eq. (66) in Ref. [29]). Therefore, in the limit $p \rightarrow 0$, a first order transition is approached, i.e., the first and second response functions converge to a discontinuous and a delta Dirac function respectively. Due to the validity of the shift closure condition, after a change of characteristic parameters, Eq. (65), the transition may be observed at an 
arbitrary value of the parameter $s$ (see Fig. 3).

In general, when the WTD involves more terms [Eq. (64)], it is not possible to find an equivalent description such as the one given by Eq. (171). Nevertheless, a similar approximated description can be formulated when the intermittence phenomenon develops.

\section{NON-RENEWAL COUNTING PROCESSES}

While the renewal processes admit a relatively simple description, a general formalism for dealing with nonrenewal counting process does not exist. In fact, the presence of memory between successive events does not admit a general description. Nevertheless, many cases can be mapped with a situation where the probability distribution for the interval between consecutive events (WTD) changes randomly after each event [13, 17].

We introduce a WTD $w\left(\tau, x_{b} \mid x_{a}\right)$ that, in contrast to the renewal case, also depends on an extra unobservable (hidden) variable $\mathbf{x}$ whose states are $\left\{x_{i}\right\}$. With $x_{a}$ and $x_{b}$ we denote the states of the hidden variable before and after an event. Then, $w\left(\tau, x_{b} \mid x_{a}\right)$ not only provides the statistics of the time intervals but also defines the probability transition between the states of $\mathbf{x}$. The most simple case corresponds to $w\left(\tau, x_{b} \mid x_{a}\right)=$ $w\left(\tau, x_{a}\right) T\left(x_{b} \mid x_{a}\right)$, where $w\left(\tau, x_{a}\right)$ is a WTD parametrized by $x_{a}$ and $T\left(x_{b} \mid x_{a}\right)$ is the transition matrix between the hidden states. Processes with memory of previous events, processes with adaptation and double stochastic processes can be covered with this approach [17]. Furthermore, a stochastic WTD may arise when describing quantum dissipative dynamics driven by classical fluctuations. In this last case $x_{a}$ and $x_{b}$ correspond to probabilities $\rho_{a}$ and $\rho_{b}$ of the initial and posterior hidden configurations [13].

By introducing a vector space spanned by the states of $\mathbf{x},\{\mid x)\}$, and by using the definition of $w\left(\tau, x_{b} \mid x_{a}\right)$, the counting probabilities can be written as

$$
P_{n}(t)=\left(1\left|\hat{P}_{n}(t)\right| \rho_{i n}\right) .
$$

Vector $\left.\mid \rho_{i n}\right)$ denotes the initial probabilities of the hidden variables, while $\left(1 \mid \equiv(1, \cdots, 1)\right.$. The operators $\hat{P}_{n}(t)$ follow from a convolution

$$
\hat{P}_{n}(t)=\int_{0}^{t} d t_{n} \int_{0}^{t_{n}} d t_{n-1} \cdots \int_{0}^{t_{2}} d t_{1} \hat{P}_{n}\left[t,\left\{t_{i}\right\}_{i=1}^{n}\right],
$$

where the "joint probability operator" reads

$$
\hat{P}_{n}\left[t,\left\{t_{i}\right\}_{i=1}^{n}\right]=\hat{P}_{0}\left(t-t_{n}\right) \hat{W}\left(t_{n}-t_{n-1}\right) \cdots \hat{W}\left(t_{1}\right) .
$$

The survival operator is $\hat{P}_{0}(t)=1-\int_{0}^{t} d t^{\prime} \hat{W}\left(t^{\prime}\right)$, and $\hat{W}(t)$ has components

$$
\left(x_{j}|\hat{W}(t)| x_{k}\right)=w\left(t, x_{j} \mid x_{k}\right) .
$$

With the previous prescriptions, it is simple to derive similar expressions for the $s$-ensemble [Eq. (17)]

$$
q_{n}(t, s)=\left(1\left|\hat{q}_{n}(t, s)\right| \rho_{i n}\right),
$$

where now the operators read

$$
\hat{q}_{n}(t, s)=\int_{0}^{t} d t_{n} \int_{0}^{t_{n}} d t_{n-1} \cdots \int_{0}^{t_{2}} d t_{1} \hat{q}_{n}\left[t,\left\{t_{i}\right\}_{i=1}^{n}, s\right],
$$

with the joint-probability operator

$\hat{q}_{n}\left[t,\left\{t_{i}\right\}_{i=1}^{n}, s\right]=\hat{q}_{0}\left(t, t_{n}, s\right) \hat{W}_{q}\left(t, t_{n-1}, s\right) \cdots \hat{W}_{q}\left(t_{1}, 0, s\right)$.

The survival operator reads $\hat{q}_{0}\left(t, t^{\prime}, s\right)=\hat{P}_{0}(t-$ $\left.t^{\prime}\right) Z\left(t^{\prime}, s\right) / Z(t, s)$, while the waiting time operator is

$$
\hat{W}_{q}\left(t, t^{\prime}, s\right)=e^{-s} \hat{W}\left(t-t^{\prime}\right) \frac{Z\left(t^{\prime}, s\right)}{Z(t, s)},
$$

The partition function reads

$$
Z(t, s)=\left(1|\hat{Z}(t, s)| \rho_{i n}\right),
$$

where its associated operator is

$$
\hat{Z}(t, s)=\sum_{n=0}^{\infty} \hat{P}_{n}(t) e^{-s n}
$$

These expressions imply that the counting process defined by the probabilities $\left\{q_{n}(t, s)\right\}_{n=1}^{\infty}$ has a similar structure to that of the set $\left\{P_{n}(t)\right\}_{n=1}^{\infty}$. In fact, the transformation of the waiting time operator [Eq. (77)] is similar to that of the renewal case [Eq. (34)]. Hence, in the long time regime, it is also possible to define a stationary waiting time operator $\hat{W}_{q}^{\infty}(\tau, s)=\lim _{t \rightarrow \infty} \hat{W}_{q}^{\infty}(t, t-$ $\tau, s)$, which reads

$$
\hat{W}_{q}^{\infty}(\tau, s)=e^{-s} \hat{W}(\tau) \exp [\tau \Theta(s)] .
$$

Thus, the non-renewal structure, in spite of the exponential decay measured by $\Theta(s)$, remains the same. Similar results to that obtained for renewal processes can be established from this last equation.

\section{SUMMARY AND CONCLUSIONS}

The LD method allows to characterize the statistics of a point process with a thermodynamic frame that is defined by the scaling rates of the counting probabilities and its characteristic function. The basis of the present analysis consists in finding an auxiliary process that maximizes the entropy function of the thermodynamic approach, Eq. (17). The conditional measurement scheme defined in Fig. 1 provides its ensemble of stochastic realizations from those of the original one. This result gives an alternative measurement interpretation of the thermodynamic frame and its conjugate counting field. Furthermore, it permits to relate the large fluctuations of a counting process with the statistical properties of a subset of its realizations.

Diverse general results were established under a renewal property, i.e., when the successive events do not develop any memory between them. We established that 
the $s$-ensemble, independently of the structure of the WTD of the counting process, is also a renewal process. An exponential decay measured by the free energy function determines its (stationary) WTD [Eq. (35)]. This function completely determines the thermodynamic formalism. In fact, a general equation for the free energy can be established in terms of its Laplace transform, Eq. (40). Similarly, the response functions follow from its normalized moments, Eq. (43).

Different phenomena that appear in the thermodynamic formalism were analyzed and derived in terms of the developed approach. We demonstrated that an invariance scale property, Eq. (44), is satisfied whenever the $s$-ensemble, in the long time regime, follows from a time rescaling of the original one, Eq. (55). Equivalently, the invariance scale property arises when the counting process can asymptotically be approximated by a renewal one defined by a Gamma distribution, Eq. (45). A shift closure property was introduced [Eq. (61)]. Its fulfillment implies that all possible statistical behaviors of the $s$-ensemble can be achieved and generated from the original one after an adequate redefinition of its characteristic parameters. Alternatively, it means that there exists a transformation of the characteristic parameters of the counting process whose sole effect is to shift all thermodynamic response functions in the $s$-direction. Different families of WTD guarantee the fulfillment of this property, Eqs. (63) and (64). Finally, we showed that renewal processes can in fact develop intermittent phenomena, where the realizations of the process and the $s$-ensemble may be characterized by intervals of high and null counting rate (Fig. 4). The properties of the response functions, which approach a first order transition, were related to the stochastic dynamics of the $s$-ensemble. While no general results can be established for non-renewal processes, we showed that similar considerations can be derived when the process is defined by a stochastic WTD, Eq. (80).

The present approach gives a solid basis for understanding the LD thermodynamic frame of a counting process. Its main advantage is the possibility of getting some general conclusions that apply to a wide class of point processes, independently of its specific structure. Extension to bidirectional counting processes, as well as non-ergodic ones may, in principle, be done along similar lines.

\section{Acknowledgments}

The author thanks fruitful discussions with E. Urdapilleta and to R.S. Echeveste for a critical reading of this manuscript. This work was supported by CONICET, Argentina, PIP 11420090100211.
[1] N.G. van Kampen, Stochastic Processes in Physics and Chemistry, (Sec. Ed., North-Holland, Amsterdam, 1992).

[2] D.R. Cox and V. Isham, Point Process, (Chapman and Hall, 1980).

[3] D.R. Cox, Renewal Theory, (Chapman and Hall, 1962).

[4] D.J. Daley and D. Vere-Jones, An Introduction to the Theory of Point Processes. Vol. I: Elementary theory and Methods, (Springer, 2003).

[5] L. Mandel and E. Wolf, Optical coherence and quantum optics (Cambridge University press, 1995).

[6] Y. Zheng and F.L. Brown, Phys. Rev. Lett. 90, 238305 (2003).

[7] Y. He and E. Barkai, Phys. Rev. Lett. 93, 068302 (2004).

[8] Y. He and E. Barkai, J. Chem. Phys. 122, 184703 (2005).

[9] Y. Zheng and F.L.H. Brown, J. Chem. Phys. 121, 7914 (2004).

[10] F. Sand and S. Mukamel, Phys. Rev. A 71, 033807 (2005).

[11] A.A. Budini, Phys. Rev. A 73, 061802(R) (2006); J. Phys. B 40, 2671 (2007); J. Chem. Phys. 126, 054101 (2007).

[12] A.A. Budini, Phys. Rev. A 79, 043804 (2009).

[13] A.A. Budini, J. Phys. B: At. Mol. Phys. 43, 115501 (2010); Phys. Rev. A 76, 023825 (2007).

[14] A. Braggio, J. König, and R. Fazio, Phys. Rev. Lett. 96, 026805 (2006).

[15] C. Flindt, T. Novotny, A. Braggio, M. Sasseti, and A. Jauho, Phys. Rev. Lett. 100, 150601 (2008).

[16] C. Flindt, T. Novotny, A. Braggio, and A. Jauho, Phys.
Rev. B 82, 155407 (2010).

[17] C. van Vreeswijk, in Analysis of Parallel Spike Trains, edited by S. Grün and S. Rotter (Springer Series in Computational Neuroscience, Springer-Verlag, Berlin, 2010).

[18] H. C. Tuckwell, Introduction to Theoretical Neurobiology, (Cambridge University Press, Cambridge, 1988).

[19] J.W. Middleton, M.J. Chacron, B. Lindner, and A. Longtin, Phys. Rev. E 68, 021920 (2003).

[20] W.H. Nesse, L. Maler, and A. Longtin, Proc. Natl. Acad. Sci. U.S.A. 107, 21973 (2010).

[21] F. Farkhooi, M.F. Strube-Bloss, and M.P. Nawrot, Phys. Rev. E 79, 021905 (2009).

[22] H. Touchette, Phys. Rep. 478, 1 (2009).

[23] J.P. Garrahan., R.L. Jack, V. Lecomte, E. Pitard, K. van Duijvendijk, and F. van Wijland, J. Phys. A 42, 075007 (2009).

[24] J. Hooyberghs and C. Vanderzande, J. Stat. Mech. P02017 (2010).

[25] V. Lecomte, C. Appert-Rolland, and F. van Wijland, J. Stat. Phys. 127, 51 (2007).

[26] R. Jack and P. Sollich, Prog. Theor. Phys. Supp. 184, 304 (2010).

[27] V. Lecomte, C. Appert-Rolland, and F. van Wijland, Phys. Rev. Lett. 95, 010601 (2005).

[28] J.P. Garrahan and I. Lesanovsky, Phys. Rev. Lett. 104, 160601 (2010); J.P. Garrahan, A.D. Armour, and I. Lesanovsky, arXiv:1103.0919v2 (2011).

[29] A.A. Budini, Phys. Rev. E 82, 061106 (2010).

[30] L.E. Reichl, A Modern Course in Statistical Physics, (J. 
Wiley and Sons, New York 2nd ed., 1998).

[31] C. Grodèche and J.M. Luck, J. Stat. Phys. 104, 489 (2001).

[32] K. Binder and D.W. Heermann, Monte Carlo Simulation in Statistical Physics: An introduction, Springer Series in
Solid-State Sciences 80 (Springer-Verlag, Berlin, 1988).

[33] K. Binder and D.P. Landau, Phys. Rev. B 30, 1477 (1984); M.S.S. Challa, D.P. Landau, and K. Binder, Phys. Rev. B 34, 1841 (1986). 